Background The person with the diagnosis and their friends/ family should be able to talk things over with a range of agencies to ask questions about the diagnosis and what it means for them. Information on help and support is available but is often poorly signposted. People with dementia (PwD) should have access to information on how to stay physically and mentally well in their own homes.

Aims Dementia hubs aim to fill this gap, providing practical information and support for PwD and their families/carers, and also for those who are worried about their memory. With clear and sensible information about recognising symptoms, research, getting help, managing financially, staying at home, treatment, being a carer and staying positive, these events will help PwD and their families to make sure that they can stay well and happy as long as possible.

Methods A 'Listening Event' was held before both Dementia Hubs commenced, with the purpose of allowing PwD and their families the chance to have their say about what support they would like to see at a new hub initiative. The Hubs provide advice/information from a wide range of organisations and individuals, eg nurses, financial planners, social workers, benefits advisers, carers support, the CQC, the Alzheimer's Society, Age UK and many others.

Results The Bay Dementia Hub which is held at St John's Hospice commenced Sept 2016 and has gone from strength to strength. Over 16 service-providers attend every month. The Fylde Coast Dementia Hub is held at Trinity Hospice has only recently started but it is hoped that it will be just as successful.

Conclusion The Hubs bring together people from many organisations and disciplines, within and beyond the hospice, to facilitate a 'one-stop shop'. To have service providers and health care support, all under one roof is quite unique!

\section{P-105 DEVELOPING A DEMENTIA POSITIVE LIVING GROUP}

Gill Austin, Pippa Wilding. St Nicholas Hospice, Bury St Edmunds, UK

\subsection{6/bmispcare-2017-hospice.131}

Background Most people diagnosed with dementia wish to maintain a 'normal life for as long as possible, thus supporting more people with dementia and their carers can help facilitate this. We decided to deliver a 'dementia positive living group' (PLG) following attendance at dementia study days and reading 'Hospice Enabled Dementia Care' Hospice UK (2015), recommending hospices develop links with other agencies and input into dementia care, focusing on education and knowledge of future/advance planning.

Aim To support those people newly diagnosed with dementia and their carers to live well with their illness. Evaluation through pre- and post- confidence surveys.

Method Our current PLG could be adapted for those diagnosed with dementia. The CCG wanted to develop dementia services, indicating the gaps were around immediately postdiagnosis. The hospice and NHS memory clinic offered the six-week PLG course. We advertised through GPs, memory clinic, social media and the hospice website. PLG offered:

- Advice and support with diagnosis

- Managing symptoms and making the most of life

- Access to resources

- future planning
Results Four couples attended. Referrals were from the memory clinic and GPs. 'Being in a group who were going through similar problems.' 'made us more positive about the future, we need this information early on the illness.' Carers reported: increased confidence caring for their relatives, sharing stories, acknowledging their experiences were normal and future planning.

Conclusions By the end of the course couples were sharing transport, arranging to meet outside of the group to support and signpost to dementia friendly venues. The next group has five couples on the waiting list. The development of enhanced dementia hospice services has enabled us to reach out to more people with dementia and work with our partners to deliver a wider service across our community.

\section{P-106 'REACHING YOU BY TEACHING YOU: IGNITING THE PASSION FOR HOSPICE-ENABLED DEMENTIA CARE'}

Helen Miller. Isabel Hospice, Welwyn Garden City, UK

10.1136/bmjspcare-2017-hospice. 132

Hospices have traditionally been seen as 'the place you go to die.' Hospice UK suggested in its 2015 guide 'Hospice enabled dementia care' that it was time to challenge this perception and share the expertise of hospices for palliative care more widely in the community because of the growing incidence and inequality of dementia care. As a result, an educational specialist/dementia lead role was created in a hospice to ignite the passion about dementia and palliative care. Dementia resources in the local community were reviewed and incorporated into a bespoke training day called 'Walking alongside dementia'.

Aim The aim was to increase understanding of dementia and its care supported by hospice and community resources for a wide range of healthcare professionals.

Methods Invitations were publicised via the hospice education programme to both primary and secondary healthcare professionals. The day covered:

- Types of dementia, diagnostics and current research

- Communication, advance care planning, identification and implementation of end-of-life care

- Collaboration and coordination with local resources

- 14 objectives were measured with a pre- and post-confidence questionnaire on a scale of 1 (low) to 5 (high).

Results In financial year 2016-2017 two sessions were originally planned. However, these quickly became oversubscribed, resulting in the need for six additional sessions. It was clear that a major need and passion had been ignited, as these additional sessions were also oversubscribed. Analysis of the eight sessions showed:

- 135 participants attended from 18 organisations with 27 different roles;

- An overall satisfaction rating for the day of 9.6 out of 10

- The average confidence level on the objectives rose from 2.5 to 4.5 , with most impact noted on community resources available.

Conclusions Collaboration transcends inequalities in palliative care and reframes the hospice role. As a team our communities have amazing power - as hospices we must strive to unlock this. 